\title{
Global fluctuations in dissipative systems
}

Cite as: AIP Conference Proceedings 1091, 201 (2009); https://

doi.org/10.1063/1.3082282

Published Online: 30 January 2009

Pablo Maynar, María Isabel García de Soria, Grégory Schehr, Alain Barrat, and Emmanuel Trizac
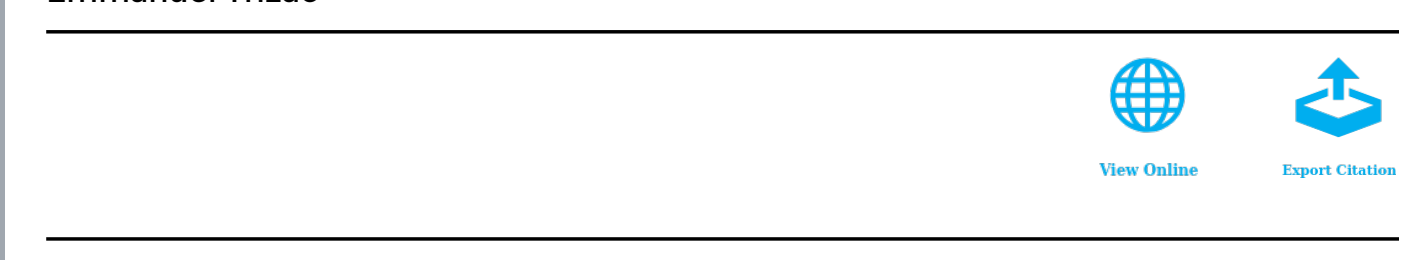

\section{Lock-in Amplifiers $\underset{\text { Watch }}{\longrightarrow}$
up to $600 \mathrm{MHz}$}

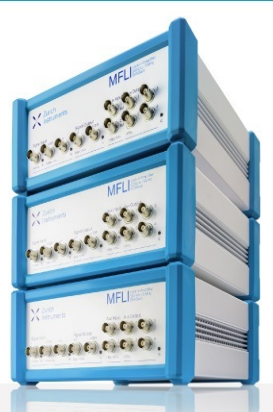




\title{
Global fluctuations in dissipative systems
}

\author{
Pablo Maynar, ${ }^{*}$, María Isabel García de Soria**, Grégory Schehr, Alain \\ Barrat and Emmanuel Trizac**
}

${ }^{*}$ Laboratoire de Physique Théorique (CNRS UMR 8627), Bâtiment 210, Université Paris-Sud, 91405 Orsay cedex, France.

${ }^{\dagger}$ Física Teórica, Universidad de Sevilla, Apartado de Correos 1065, E-41080, Sevilla, Spain

** Université Paris-Sud, LPTMS, UMR 8626, Orsay Cedex, F-91405 and CNRS, Orsay, F-91405

¿Laboratoire de Physique Théorique (CNRS UMR 8627), Bâtiment 210, Université Paris-Sud, 91405 Orsay cedex, France

\begin{abstract}
Our goal here is to investigate the influence of velocity correlations on the fluctuations of several global quantities for two different systems. In the first case, we study the fluctuations of the total number of particles, momentum and energy in a probabilistic ballistic annihilation model. For this model, when there is a binary encounter, the particles annihilate with certain probability. In the second case, we analyze the fluctuations of the total energy in a granular system which is driven by a stochastic thermostat. The theoretical analysis is based on the study of the equation for the two-particle distribution function. The predictions are in very good agreement with simulations results.
\end{abstract}

Keywords: Dissipative system, granular flow, kinetic theory, global fluctuations.

PACS: $51.10 .+\mathrm{y}, 05.20 . \mathrm{Dd}, 82.20 . \mathrm{Nk}$

\section{INTRODUCTION}

In the last years, there has been some interest in the study of the microscopic and macroscopic properties of granular media. It has been realized that inelasticity, which is the main feature of grain interactions, gives rise to a very interesting phenomenology [1]. One of the simplest model that is usually used is the inelastic hard sphere (IHS) model, which consists of an ensemble of $N$ hard particles of mass $m$ and diameter $\sigma$ that collide inelastically. Then, if $\mathbf{v}_{1}$ and $\mathbf{v}_{2}$ are the velocities of two particles before a collision, the velocities after the collision read

$$
\mathbf{v}_{1}^{\prime}=\mathbf{v}_{1}-\frac{1+\alpha}{2}\left[\left(\mathbf{v}_{1}-\mathbf{v}_{2}\right) \cdot \hat{\sigma}\right] \hat{\sigma}, \quad \mathbf{v}_{2}^{\prime}=\mathbf{v}_{2}+\frac{1+\alpha}{2}\left[\left(\mathbf{v}_{1}-\mathbf{v}_{2}\right) \cdot \hat{\sigma}\right] \hat{\sigma},
$$

where $0 \leq \alpha \leq 1$ is the coefficient of normal restitution ( $\alpha=1$ is the elastic limit) and $\hat{\sigma}$ is a unit vector joining the centers of particles at contact.

On the other hand, systems where particles may react, change chemical or physical nature and ultimately disappear, model also a rich variety of phenomena. When reactions are controlled ballistically, the system can be modeled by the so-called probabilistic ballistic annihilation (PBA) model which is an assembly of hard spheres or disks which annihilate with probability $p$ or collide elastically (formula (1) with $\alpha=1$ ) with probability $1-p$ every time two particles collide [2].

Theoretically, one of the tools used to understand the rich phenomenology of these systems is kinetic theory, which is extended naturally by the "inelastic collision rule"

CP1091, Modeling and Simulation of New Materials: Tenth Granada Lectures

edited by Pedro L. Garrido, Pablo I. Hurtado, and Joaquin Marro

(c) 2009 American Institute of Physics 978-0-7354-0624-7/09/\$25.00 
previously introduced. In the granular case the number of particles and momentum are conserved but the energy is not, while in the PBA case there are no collisional invariants. Within the framework of the PBA and the granular model, most of the work carried out up to now has focused on the kinetic equations for the one-body distribution function and the information following from them $[1,2]$. In this work we go further and we analyze the role of the correlations in the fluctuations of the most relevant global quantities for both systems.

\section{KINETIC THEORY PREDICTIONS}

Let us analyze first the case of the PBA model. The hierarchy of equations for the reduced distribution functions can be shown to be similar to the one describing elastic collisions, but replacing the binary elastic collision operator, $T_{e}\left(\mathbf{v}_{1}, \mathbf{v}_{2}\right)$, by the operator describing annihilating collisions, $T_{p}\left(\mathbf{v}_{1}, \mathbf{v}_{2}\right)$, which depend explicitly on $p$ [3]. Assuming molecular chaos, i.e. that no correlations exist between colliding particles, the equation for the one-particle distribution function $f_{1}\left(x_{1}, t\right)$ is the Boltzmann equation

$$
\left[\frac{\partial}{\partial t}+L\left(x_{1}\right)\right] f_{1}\left(x_{1}, t\right)=\int d x_{2} \delta\left(\mathbf{r}_{12}\right) T_{p}\left(\mathbf{v}_{1}, \mathbf{v}_{2}\right) f_{1}\left(x_{1}, t\right) f_{1}\left(x_{2}, t\right)
$$

where $x \equiv\{\mathbf{r}, \mathbf{v}\}$ is a field variable and $L\left(x_{1}\right)=\mathbf{v}_{\mathbf{1}} \cdot \frac{\partial}{\partial \mathbf{r}_{1}}$.

The equation for the correlation function $g_{2}\left(x_{1}, x_{2}, t\right) \equiv f_{2}\left(x_{1}, x_{2}, t\right)-f_{1}\left(x_{1}, t\right) f_{1}\left(x_{2}, t\right)$, where $f_{2}$ is the two-particle distribution function, can be obtained in the same spirit, by neglecting the three-body correlations

$$
\begin{array}{r}
{\left[\frac{\partial}{\partial t}+L\left(x_{1}\right)+L\left(x_{2}\right)-K\left(x_{1}, t\right)-K\left(x_{2}, t\right)\right] g_{2}\left(x_{1}, x_{2}, t\right)} \\
=\delta\left(\mathbf{r}_{12}\right) T_{p}\left(\mathbf{v}_{1}, \mathbf{v}_{2}\right) f_{1}\left(x_{1}, t\right) f_{1}\left(x_{2}, t\right)
\end{array}
$$

where we have introduced the linear operator $K\left(x_{i}, t\right)$ which is also a functional of the PBA binary collision operator [3].

There is a special solution of these equations which corresponds to an homogeneous isotropic system in which all the time dependence in the distribution functions is embodied in the temperature and density (the second velocity moment of $f_{1}$ ). This is the reference state (in the sense that for a wide class of initial conditions the system reaches this state in the long time limit) and it is called the Homogeneous Decay State (HDS) $[2,3]$. In this case, assuming some properties concerning the hydrodynamic part of the spectrum of the operator $K[3]$, it is possible to show that the second moment of the fluctuations of the total number of particles $\delta N=N-N_{H}$, the total momentum $\delta P_{i}=P_{i}$ and total energy $\delta E=E-E_{H}$ scale as

$$
\left\langle\delta N^{2}(t)\right\rangle \propto N_{H}(t), \quad\left\langle\delta P_{i}^{2}(t)\right\rangle \propto E_{H}(t), \quad\left\langle\delta E^{2}(t)\right\rangle \propto E_{H}^{2}(t) / N_{H}(t),
$$

where $N_{H}(t)$ and $E_{H}(t)$ are the mean values of the total number of particles and total energy respectively in the HDS. The constants of proportionality are explicitly known 
as some integrals of the correlation function and can be rewritten in terms of the oneparticle distribution function [3] due to equation (3). This shows the important role of velocity correlations in the fluctuations of global quantities.
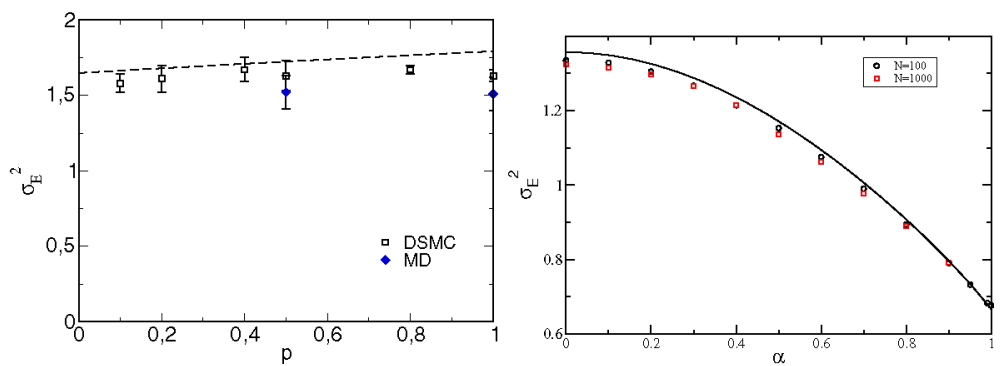

FIGURE 1. Scaled second moment of the energy fluctuations for a PBA system (left panel) as a function of $p$ and for a heated granular system (right panel) as a function of $\alpha$. The lines are the theoretical predictions and symbols are results from simulations.

Now, let us discuss very briefly the granular case. The system is heated uniformly by adding a random velocity to the velocity of each particle at equal times in such a way that the time between random kicks is small compared to the mean free time. Then, between collisions, the velocities of the particles change many times due to the thermostat. In the dilute limit, assuming molecular chaos and that the sizes of the jumps due to the thermostat are small compared to the scale in which the distribution varies, we obtain similar equations to the one obtained for the PBA system, but as functionals of the inelastic binary collision operator, $T_{\alpha}\left(\mathbf{v}_{1}, \mathbf{v}_{2}\right)$. The only difference is that some new terms appear (they are essentially diffusive Fokker-Planck terms) as a consequence of the thermostat. In this case the reference state is stationary and the total number of particles and total momentum are conserved. The fluctuations of the total energy can be evaluated in a similar way, which provides an explicit formula for the variance of the fluctuations.

In the Figure, we have plotted the theoretical prediction for the scaled variance of the total energy, $\sigma_{E}^{2} \equiv N_{H}\left\langle\delta E^{2}\right\rangle / E_{H}^{2}$ (lines) for the two systems and the points are results from Molecular Dynamic (MD) and from the Direct Monte Carlo method (DSMC). The simulations for the granular system come from the reference [4]. As we can see, the agreement is quite good showing that the presence of velocity correlations are important in order to understand these fluctuations.

\section{REFERENCES}

1. I. Goldhirsch, Annu. Rev. Fluid Mech. 35, 267 (2003).

2. F. Coppex, M. Droz, and E. Trizac, Phys. Rev. E 69, 011303 (2004).

3. P. Maynar, M. I. García de Soria, G. Schehr, A. Barrat, and E. Trizac, Phys. Rev E 77, 051128 (2008).

4. P. Visco, A. Puglisi, A. Barrat, F. van Wijland, and E. Trizac, Eur. Phys. J. B 51, 377 (2006) 